2. Gruber PJ, Fuller S, Cleaver KM, Abdullah I, Gruber SB, Nicholson SC, et al. Early results of single-stage biventricular repair of severe aortic hypoplasia or atresia with ventricular septal defect and normal left ventricle. J Thorac Cardiovasc Surg. 2006;132: 260-3.

3. Ohye RG, Kagisaki K, Lee LA, Mosca RS, Goldberg CS, Bove EL. Biventricular repair for aortic atresia of hypoplasia and ventricular septal defect. J Thorac Cardiovasc Surg. 1999;118:648-54.
4. Bacha EA, Daves S, Hardin J, Abdulla R, Anderson J, Kahana M, et al. Single-ventricle palliation for high risk neonates: the emergence of an alternative hybrid stage I strategy. J Thorac Cardiovasc Surg. 2006; 131:163-71.

5. Michel-Behnke I, Akintuerk H, Marquardt I, Mueller M, Thul J, Bauer $\mathrm{J}$, et al. Stenting of the ductus arteriosus and banding of the pulmonary arteries: basis for various surgical strategies in newborns with multiple left heart obstructive lesions. Heart. 2003;89:645-50.

\title{
Anomalous origin of the left main artery from the pulmonary artery: Adult presentation with systemic collateral supply and giant right coronary artery aneurysm
}

Pietro Bajona, MD, ${ }^{a}$ Daniele Maselli, MD, ${ }^{\text {a }}$ Roberto Dore, MD, ${ }^{\mathrm{b}}$ and Gaetano Minzioni, MD, ${ }^{a}$ Pisa and Pavia, Italy

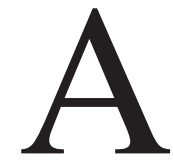

nomalous origin of the left coronary artery (LCA) from the pulmonary artery (ALCAPA) in adulthood is rare, and clinical presentation depends on collateral circulation between and to the coronary arteries. We present an adult patient with ALCAPA with systemic collateral supply to the LCA and a giant right coronary artery (RCA) aneurysm who was successfully treated by means of closure of the anomalous origin of the left main artery and repair of the aneurysm.

\section{Clinical Summary}

A 44-year-old male physician was referred to our hospital because of a 6-month history of dizziness and increasing dyspnea on exertion. He had been a rugby player since childhood and had played semiprofessionally until a year before. He smoked 10 cigarettes per day, and he had no other risk factors for coronary artery disease. The results of physical examination were normal. Blood pressure was within normal limits. Laboratory test results were normal. Chest radiography revealed the presence of a moderate cardiomegaly and opacity overlapping the right heart border and the right pulmonary hilum. Electrocardiography revealed sinus

From the Cardiothoracic Department, ${ }^{a}$ Azienda Ospedaliera Universitaria Pisana, Pisa, Italy; and the Department of Radiology, ${ }^{\mathrm{b}}$ University Hospital of Pavia, Pavia, Italy.

Received for publication March 26, 2007; accepted for publication April 20, 2007.

Address for reprints: Pietro Bajona, MD, Cardiothoracic Department, Azienda Ospedaliera Universitaria Pisana, Via Paradisa 2, 56124 Pisa, Italy (E-mail: pietro.bajona@libero.it).

J Thorac Cardiovasc Surg 2007;134:518-20

$0022-5223 / \$ 32.00$

Copyright $\odot 2007$ by The American Association for Thoracic Surgery doi:10.1016/j.jtcvs.2007.04.016 rhythm and left ventricular hypertrophy with normal patterns of PR, QT, and ST segment.

Transthoracic echocardi-

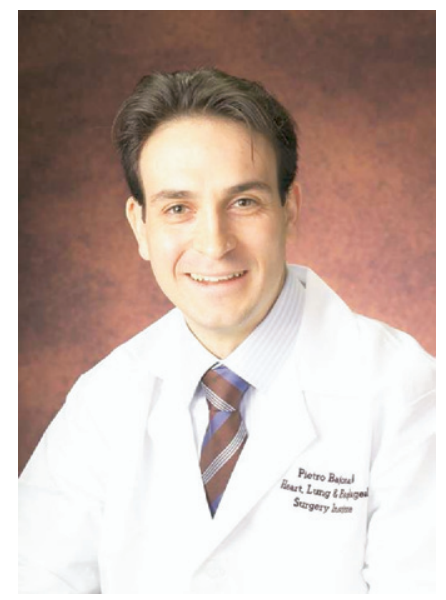

Dr Bajona ography showed a $7.0 \times$

$9.0-\mathrm{cm}$ aneurysm involving the proximal RCA and wide ectasia of the coronary arteries. At Doppler scanning, a fistula between the left main artery and the main pulmonary artery (MPA) was observed.

Coronary angiography showed that the LCA was supplied through aneurysmal collateral branches from the RCA and from both anomalous branches of the right internal thoracic artery (RITA) and bronchial artery (BA; Figure 1). Retrograde filling of the LCA and MPA was observed by injecting the distal RCA. Chest computed tomographic scanning with contrast demonstrated a large aneurysm of the proximal portion of the RCA. The distal RCA was dilated and tortuous (Figure 2). At the time of the operation, a giant RCA aneurysm and a dilated and tortuous anterior interventricular artery were found. The aneurysm was located laterally to the ascending aorta and anterior to the superior vena cava. Both abnormal branches from the RITA and BA were not visible. Hypothermic $\left(26^{\circ} \mathrm{C}\right)$ right femorofemoral cardiopulmonary bypass was established. The ascending aorta was clamped, and antegrade, cold, intermittent blood cardioplegia was delivered into the aortic root.

The MPA was transected just above the sinotubular junction. Massive backflow originating from the RCA and RITA and BA branches was observed draining into the MPA. Cardiopulmonary bypass was arrested for 2 minutes to permit the identification and ligation of the LCA ostium with a 4-0 polypropylene running suture.

The RCA aneurysm was transected and replaced with a 3-cmlong segment of an 8-mm-diameter Dacron graft. After patient rewarming, deairing was performed, the aortic crossclamp was released, and the operation was completed in a routine fashion. 


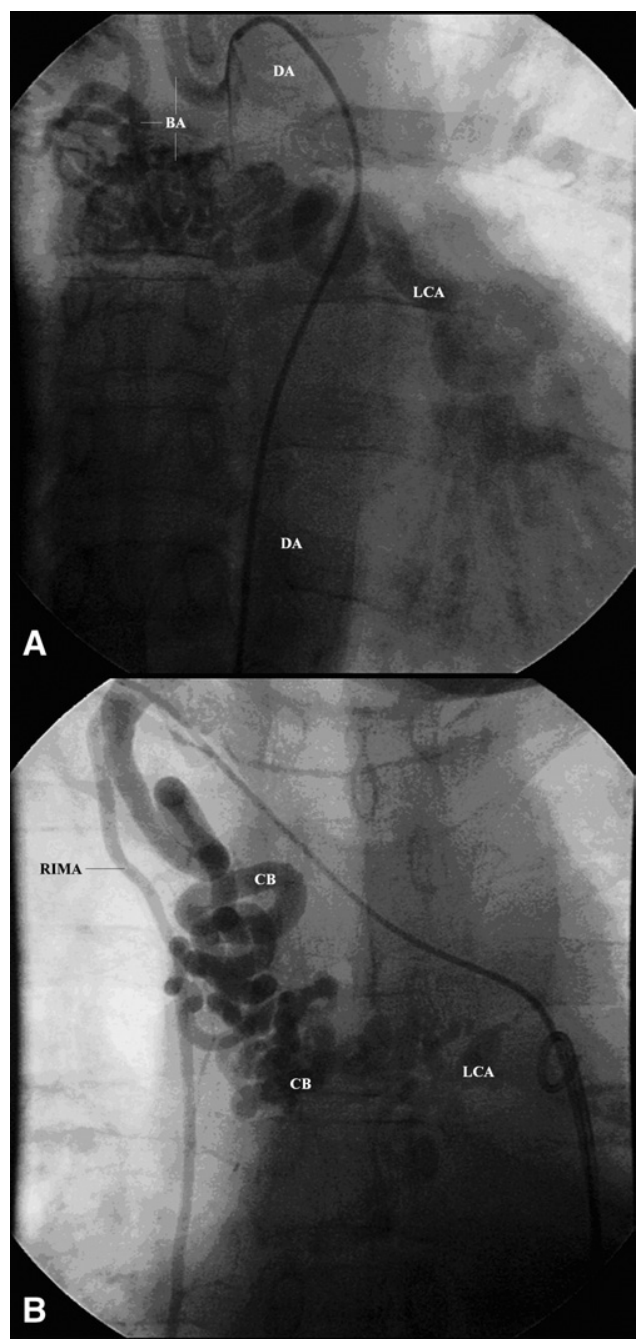

Figure 1. A, Dye injection into the descending aorta showing a bronchial artery connected through an anomalous course with the left coronary artery. B, Dye injection into the right internal thoracic artery showing an abnormal collateral branch connecting to the left coronary artery. $D A$, Descending aorta; $B A$, bronchial artery; $L C A$, left coronary artery; RITA, right internal thoracic artery; $C B$, collateral branch.

Intraoperative transesophageal echocardiography showed a normal left ventricular volume with a normal ejection fraction. The postoperative course was uneventful. Pathologic examination of the resected RCA aneurysm revealed atherosclerotic change and chronic inflammatory tissue. Chest computed tomography was performed 10 months postoperatively and showed optimal reconstruction of the RCA.

At the 1-year follow-up, the patient was found to be symptomfree without medication.

\section{Discussion}

ALCAPA is a rare congenital anomaly that is often referred to as Bland-White-Garland syndrome. ${ }^{1}$ It occurs in approximately 1 in

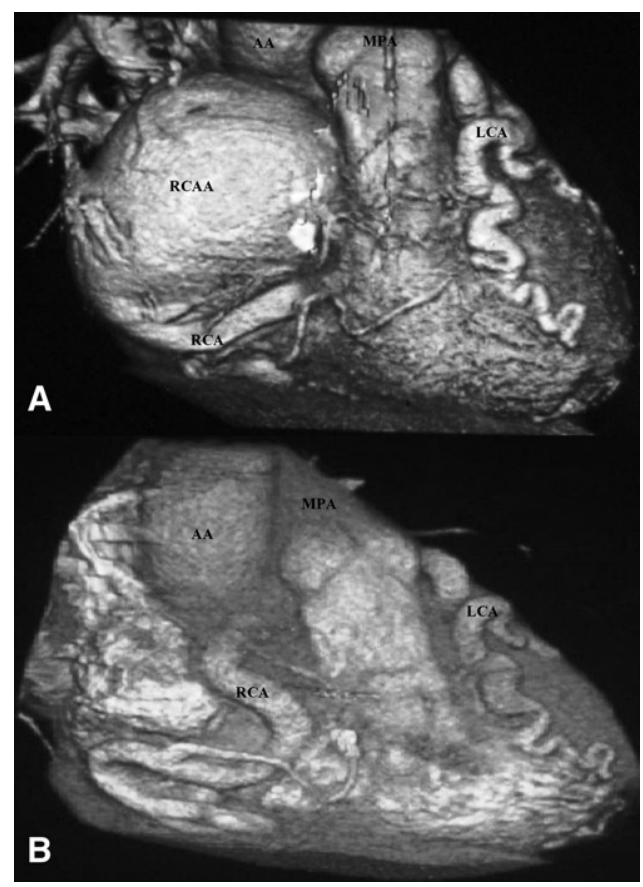

Figure 2. A, Preoperative computed tomographic scan showing a giant aneurysm involving the proximal right coronary artery and wide ectasia of the coronary arteries. B, Ten months' follow-up computed tomographic scan showing optimal reconstruction of the right coronary artery. $A A$, Ascending aorta; MPA, main pulmonary artery; $L C A$, left coronary artery; $R C A A$, right coronary artery aneurysm; $\boldsymbol{R C A}$, right coronary artery.

300,000 children. Most infants die within the first year of life without treatment. Survival beyond infancy depends on the development of adequate collateral circulation from the RCA or from another source to the LCA. However, even in the patients who survive to adulthood, sudden death frequently occurs. ${ }^{2}$ Surgical intervention is recommended in all patients, even in asymptomatic adults with no objective evidence of ischemia. Most patients will have significant RCA dilatation. ${ }^{3}$ This is likely related to the long-standing increased flow related to the left anomalous coronary artery, which, perhaps, increases the diameter over the course of time. However, a huge RCA associated with ALCAPA is an unusual presentation. ${ }^{4}$ The asymptomatic course of our case seems to be related to an optimal balance between collateral flow from the RCA and systemic blood supply from the RITA and BA branches. There are 4 similar cases describing ALCAPA with possible systemic collateral supply, but only in one of them was supply from the BA confirmed. ${ }^{5}$ To the best of our knowledge, there are no described cases with blood supply from RITA branches. In this case, a simple ligation was performed to avoid coronary flow imbalance and thrombosis in the dilated coronary arteries and because of the patient's well-developed systemic collateral arteries. The present observation is unique in that it describes a case of ALCAPA with LCA circulation supplied by two 
abnormal systemic branches and a huge RCA aneurysm in adulthood.

\section{References}

1. Bland EF, White PD, Garland J. Congenital anomalies of the coronary arteries: report of an unusual case associated with cardiac hypertrophy. Am Heart J. 1933;8:787-801

2. Wesselhoeft H, Fawcett JS, Johnson AL. Anomalous origin of the left coronary artery from the pulmonary trunk. Its clinical spectrum, pathology, and pathophysiology based on a review of 140 cases with seven further cases. Circulation. 1968;38:403-25.
3. Koike K, Musewe NN, Smallhorn JF, Freedom RM. Distinguishing between anomalous origin of the left coronary artery from the pulmonary trunk and dilated cardiomyopathy: role of echocardiographic measurement of the right coronary artery diameter. Br Heart J. 1989;61: 192-7.

4. Arsan S, Naseri E, Keser N. An adult case of Bland White Garland syndrome with huge right coronary aneurysm. Ann Thorac Surg. 1999; 68:1832-3.

5. Barbetakis N, Efstathiou A, Papagiannopoulou P, Soulountsi V, Fessatidis I. A long-term survivor of Bland-White-Garland syndrome with systemic collateral supply: a case report and review of the literature. BMC Surg. 2005;5:23.

\section{Acute intramural hematoma of the aorta as a cause of positive fluorodeoxyglucose positron emission tomography/computed tomography}

Alex Ryan, MD, Barry McCook, MD, Biatta Sholosh, MD, Daniel A. Pryma, MD, Eric Jablonowski, Carl Fuhrman, MD, and Todd M. Blodgett, MD, Pittsburgh, Pa

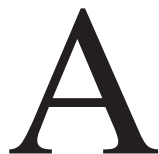

cute intramural hematoma (IMH) is a process characterized by aortic wall hemorrhage without intimal disruption. It has recently been added to the list of acute aortic syndromes (AASs), which also includes aortic dissection (AD), penetrating atherosclerotic ulcer (PAU), aneurysmal leak, and traumatic transection. IMH accounts for roughly $10 \%$ to $30 \%$ of AAS cases. ${ }^{1}$

The hemorrhage of IMH is said to arise from (1) rupture of the vasa vasorum in the adventitia of the aortic wall and (2) progression of a PAU. PAU is largely a disease of the descending aorta, making vasa vasorum rupture the major cause of upper aorta, type A IMH. ${ }^{2}$ These type A lesions usually occur within a few centimeters of the aortic valve on the lateral wall of the ascending aorta. The wall failure in IMH occurs nearer the adventitia than does the failure of $\mathrm{AD}$, which helps explain both the higher incidence of rupture $(35 \%)^{3}$ and the lower likelihood of reentry, which is the cause of false lumen flow in AD. Progression to AD is nevertheless not uncommon during follow-up of IMH cases, with a prevalence of $12 \%{ }^{4}$

Clinical presentation of AAS classically involves sudden back pain, chest pain, or both, and it generally presents in hypertensive patients $(85 \%) .{ }^{5}$ AAS can be mistaken for myocardial infarction with cardiogenic shock, especially when rupture leads to tamponade. Traumatic deceleration injuries can cause IMH through in-

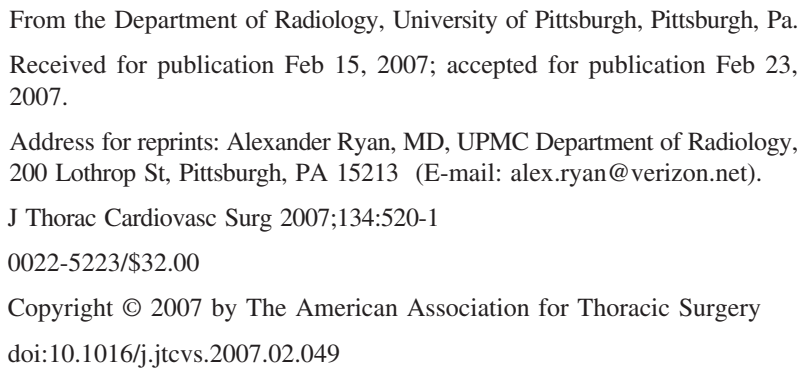

complete traumatic aortic rupture. Misdiagnosis in these patients is often fatal, making early intervention essential.

IMH can be diagnosed using computed tomography (CT) and usually appears as a crescent-shaped area that is higher attenuation than intraluminal blood on noncontrast images (Figure 1). To our knowledge, IMH has not been described in the literature as a cause of fluorodeoxyglucose (FDG) uptake on FDG positron emission tomography (PET) or PET/CT.

\section{Clinical Summary}

Initial PET/CT imaging was performed on a 70-year-old woman for staging of colorectal cancer. PET and diagnostic unenhanced CT images were acquired from the base of the skull to the proximal thighs on a GE Discovery LS PET/CT (GE Medical Systems, Waukesha, Wis) approximately 60 minutes after intravenous injection of $540 \mathrm{MBq}$ of FDG. The PET image showed a subtle tiny focus of FDG activity in the aorta; however, no correlative CT abnormality could be identified to make a definitive diagnosis, and the uptake was attributed to atherosclerotic change (Figure 2).

Approximately 6 months later, PET/CT was performed for restaging after partial colectomy. Contrast CT was not obtained, but on unenhanced CT, 2 new findings were found: mild aneurysmal dilation of the ascending aorta and a subtle but enlarging, high-attenuation, crescent-shaped area along the lateral aortic wall.

On the PET portion of the examination, findings included a crescent-shaped area of intense increased FDG activity along the left lateral wall of the ascending aorta with a maximum standardized uptake value (SUV) of 6.4. These findings confirmed the diagnosis of acute IMH. The focus noted in the earlier study was now recognized as a very early stage of this patient's IMH.

\section{Discussion}

Only recently was IMH differentiated from $\mathrm{AD}$ as an entity with its own characteristics. It has in common with $\mathrm{AD}$ its relation to hypertension and the unsolved dilemma regarding whether to 